\title{
齿根三维粗粘表面的应力集中系数计算研究
}

\author{
何玉辉 $^{1}$ 胡 航 $^{1}$ 唐进元 $^{1}$ 周 炜 $^{2}$ \\ (1. 中南大学高性能复杂制造国家重点实验室 长沙 410083; \\ 2. 湖南科技大学难加工材料高效精密加工湖南省重点实验室 湘潭 411201)
}

\begin{abstract}
摘要: 考虑粗粘表面影响因素的齿轮齿根处应力集中系数计算是齿轮弯曲疲劳寿命精准预估的难题, 以磨削喷丸后的直齿轮 为研究对象, 研究粗粘表面下的齿根应力与应力集中计算问题。采用白光干涉仪 Wyko NT9100 对磨削喷丸齿根粗楉表面进 行测量, 得到粗粘表面形貌数据, 基于空间坐标变换原理, 使用 Python 对有限元软件进行二次开发, 通过调整齿根细化网格 节点坐标实现了齿根表面粗糙形貌的添加工作, 建立了齿根过渡曲面三维粗徏表面有限元模型并进行仿真分析。通过数值计 算得到三维粗粘表面参数下的齿根应力分布与应力集中系数, 对粗精表面参数与应力集中系数的关联规律进行非线性回归分 析, 建立粗粘表面参数与应力集中系数的关联规律。结果表明, 粗粘表面参数 $S_{a} 、 S_{v} 、 S_{10 z}$ 拟合应力集中系数得到拟合公 式的相关系数分别为 $0.799,0.784,0.914$, 十点区域高度参数 $S_{10 z}$ 能较好地表征齿根表面的应力集中。
\end{abstract}

关键词: 齿根; 粗糙表面; 应力集中系数; 计算方法; 喷丸

中图分类号: TH132

\section{Stress Concentration Calculation Factor of Tooth Root with 3D Rough Profile}

\author{
HE Yuhui $^{1}$ HU Hang ${ }^{1}$ TANG Jinyuan ${ }^{1}$ ZHOU Wei ${ }^{2}$ \\ (1. State Key Laboratory of High Performance Complex Manufacturing, Central South University, Changsha \\ 410083; \\ 2. Hunan Provincial Key Laboratory of High Efficiency and Precision Machining of Difficult-to-Cut Material, \\ Hunan University of Science and Technology, Xiangtan 411201)
}

\begin{abstract}
The calculation of the stress concentration factor in the tooth root of the gear considering the influence of the rough surface is a difficult problem in accurately predicting the bending fatigue life of the gear. The ground and shot peened spur gear as the research object to study the root stress and stress concentration under the rough surface of tooth root. The white light interferometer Wyko NT9100 is used to measure the rough surface of the ground and shot peened tooth root, and the rough surface topography data is obtained. Based on the principle of space coordinate transformation, the finite element software is redeveloped by using Python, adjusting the coordinates of the tooth root refined mesh nodes, the rough topography of the tooth root surface is added, and the 3D rough surface finite element model of the tooth root transition surface is established and simulated. Through numerical calculation, the root stress distribution and stress concentration factor under 3D rough surface parameters are obtained, and the correlation law between rough surface parameters and stress concentration factor is performed nonlinear regression analysis, and the correlation law between rough surface parameters and stress concentration factor is established. The results show that the correlation coefficients of the fitting formulas obtained by fitting the rough surface parameters $S_{a}, S_{v}, S_{10 z}$ and stress concentration coefficients are $0.799,0.784$, 0.914, respectively. The ten-point region height parameter can better characterize the stress concentration on the tooth root surface.
\end{abstract}

Key words: gear tooth root; rough surface; stress concentration factor; method of calculation; shot peen

\footnotetext{
* 国家重点研发计划(2018YFB2001300)、国家自然科学基金青年基金(51705142) 和高性能复杂制造国家重点实验室自主研究课题(ZZYJKT2018)资助项目。 20200813 收到初稿, 20210121 收到修改稿
} 


\section{0 前言}

齿轮作为传动系统的核心部件, 被广泛运用于 航空航天、汽车坦克和高精密仪器制造等领域, 喷 丸强化是高性能齿轮制造的关键工序, 通过齿根喷 丸强化引入残余压应力可以有效提高齿轮使用寿 命。喷丸与其他机械加工方法一样, 最终零件表面 是凹凸不平的微观形貌, 这些微观形貌的谷底区域 在工件工作时会产生应力集中, 表面粗粘度越大, 产生的应力集中越严重, 工件的疲劳寿命下降越快 [1]。因此, 精确计算齿根喷丸粗楉表面的应力与应 力集中系数对评估齿轮弯曲疲劳寿命极为关键。

对于粗糙表面与应力集中系数的关联性研究, $\mathrm{NEUBER}^{[2]}$ 将粗糙表面轮廓近似为连续相邻的缺口, 并由缺口深度和宽度是影响缺口应力集中的主要因 素, 推广到基于粗䊁表面参数 $R_{z}$ 和谷底曲率半径 $\rho$ 的应力集中系数计算公式。AROLA 等 ${ }^{[3]}$ 依据 NEUBER 提出的经验公式建立了 Arola-Ramulu 模 型, 将实际的表面形貌近似为连续相邻的正弦状缺 口展开研究, 并通过实验验证了该模型的准确性。 $\mathrm{CHAN}^{[4]}$ 对电子束加工得到的板块表面进行测量, 研究了具有凹痕特征的形貌的应力集中, 提出了加 工制造过程中对表面形貌的要求; KANTZOS ${ }^{[5]}$ 借助 神经网络, 将应力集中和表面形貌进行关联分析, 实现了裂纹萌生的预测。在数值计算方面, 学者们 通过对具有特定规则的形貌进行简化研究, 采用一 阶边界摄动法, 基于 Hilbert 变换和能量守恒原理, 预测了拉伸作用下二维表面形貌上的应力分布并进 行实验验证 ${ }^{[6-10]}$ 。然而, 目前可见文献对于粗粘表 面与应力集中系数的关联规律大多基于二维表面形 貌展开研究, 对于三维实测表面形貌引起的应力集 中研究较少, MEREUTA ${ }^{[11]}$ 分别将 Neuber 模型和 Arola-Ramulu 模型的公式中二维粗糙表面参数转换 为对应的三维粗䊁表面参数进行计算, 并经过试验 验证了修正公式的可靠性。 $\mathrm{LI}^{[12]}$ 基于表面形貌重构 技术对重构表面引起的应力集中进行分析, 利用少 数几个重构平面模型分析了粗粘表面参数 $S_{a}$ 疲劳 寿命之间的关系。XU $\mathrm{XU}^{[13]}$ 研究了腐蚀对 Q235 钢板疲 劳寿命的影响, 借助 ANSYS 软件生成了三维粗粘 形貌并分析了三种腐蚀坑与疲劳寿命的关系, 最后 与疲劳实验进行对比验证。在 GB/T 3480-1997 中 通过大量的疲劳实验提出了相对齿根表面状况系数 经验公式 ${ }^{[14]}$, 然而该经验公式没有给出粗粘度表面 应力集中系数的计算表达式, 而是通过相对齿根应
力集中系数的比值拟合的公式间接获得应力集中对 齿轮弯曲疲劳寿命的影响, 且该经验公式仅用 1 个 二维粗粘度参数 $R_{z}$ 进行表征, 显然无法精确评估三 维实际表面产生的应力集中。

随着对三维表面的认识, 用于表征粗糙表面形 貌特征的三维粗粘度参数多达几十个，不同的粗粘 度参数表征表面的不同性能，而对于表征应力集中 的三维粗粘度参数, 目前尚未得到共识。且无论是 二维还是三维粗䊁表面问题, 现阶段所有研究对象 均是以平板或者简化为平面模型进行研究, 解决齿 根粗糙表面下实际应力精确计算, 得到表征粗粘表 面应力集中合适的形貌参数问题是高端齿轮、高性 能齿轮设计制造的核心技术之一，具有较大的理论 与工程应用价值。

论文以磨削后喷丸的直齿轮为研究对象, 提取 齿轮齿根三维粗䊁表面形貌信息, 依据空间坐标变 换原理, 研究齿根过渡曲面添加三维粗鋉面的建模 方法, 从而突破现有齿轮强度分析只能创建光滑表 面模型的限制, 建立基于实测三维表面形貌的齿根 区域有限元模型, 对有限元计算得到的数据进行非 线性曲线拟合, 得到齿根三维粗糙表面参数与应力 集中系数 $K_{t}$ 的关联公式, 为后续的齿轮弯曲疲劳寿 命精准预测和齿轮表面完整性参数设计提供相应的 参考。

\section{1 齿根表面形貌测量}

为研究齿根喷丸表面危险区域的表面粗䊁度与 应力集中系数的关系, 首先确定齿根危险区域的具 体位置, 基于磨削喷丸加工的齿轮(表 1), 借助 Matlab 编程生成直齿轮齿廓, 将齿廓导入 CATIA, 通过阵列、拉伸等操作生成直齿轮实体模型, 将齿 轮实体模型导入 ABAQUS 中进行准静态仿真分析, 查看仿真结果应力云图, 确定了齿根危险区域 ${ }^{[15]}$ (应 力较大区域)。

表 1 齿轮参数

\begin{tabular}{cc||cc}
\hline 齿轮参数 & 数值 & 齿轮参数 & 数值 \\
\hline 模数 $/ \mathrm{mm}$ & 3 & 齿顶间隙系数 & 0.25 \\
齿数 & 29,49 & 压力角 $\left./{ }^{\circ}\right)$ & 25 \\
齿宽 $/ \mathrm{mm}$ & 20 & $\begin{array}{c}\text { 变位系数 } \\
\text { 大齿传递的转矩 } \\
/(\mathrm{N} \cdot \mathrm{m})\end{array}$ & 0 \\
齿顶高系数 & 1 & 239 \\
\hline
\end{tabular}

其次, 测量表面形貌。本文借助 ABAQUS 研 究齿根危险区域表面粗粘度对应力集中的影响, 通 过调整网格节点坐标值实现粗粘度的添加，而齿根 
危险区域的网格数量取决于形貌测量的采样点数, 因此, 选择较为合适的采样间隔减小网格数量、提 高仿真效率是很有必要的。文中采用白光干涉仪 Wyko NT9100 对粗粘形貌进行测量。对于表面形貌 的测量, 采样间隔越小, 表面形貌还原越完整。考 虑文中对有限元网格数量的要求, 在保留形貌基本 特征前提下, 适当选择较大的采样间隔, 舍弃部分 形貌信息。一般地, 采样频率大于形貌频率 4 倍时, 采样离散数据点可以基本复原形貌的大致轮廓 特征 $^{[16]}$ 。

文中以喷丸后的粗糙表面为研究对象, 主要研 究喷丸处理后产生的丸坑形貌对应力集中的影响。

一般地, 表面形貌的应力集中最有可能产生在粗粘 形貌最深的几个谷底区域之一, 已知齿轮喷丸直径 约为 $120 \mu \mathrm{m}$, 喷丸后产生的几个最深丸坑深度平均 值绝大部分在 $5 \mu \mathrm{m}$ 以上(详细数据见表 2 中的 $S_{10 z}$ ), 则丸坑的表面直径大于 $40 \mu \mathrm{m}$ 。因此, 在满足采样 频率大于 4 倍的形貌频率(丸坑表面直径)的基础上, 论文测量时选择采样间隔为 $10 \mu \mathrm{m}$, 采样面积 为 $1 \mathrm{~mm} \times 1 \mathrm{~mm}$ 。得到磨削后喷丸表面形貌如图 1 所示。

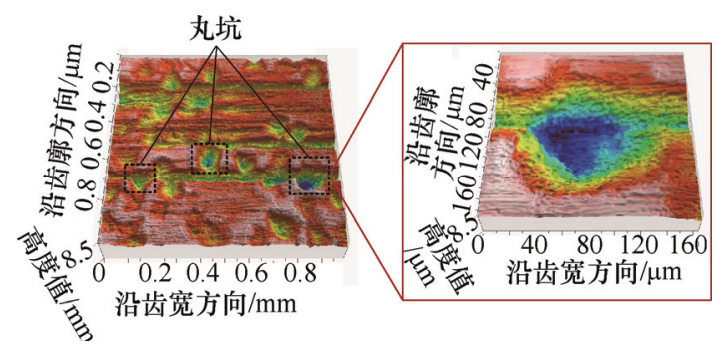

图 1 齿轮磨削喷丸后表面形貌

\section{2 考虑三维粗粘度的齿根应力有限元 计算}

\section{1 齿轮网格划分及细化}

齿根应力有限元计算需要精确的齿根形貌与齿 面形貌, 渐开线齿面的计算按渐开线方程计算 ${ }^{[17-18]}$, 齿根过渡曲面的构建按课题组前期提出的建模方法 构建, 详细齿根过渡曲面构建方法可参考文 献[19-20]。

本文通过调整网格节点坐标实现粗鉌度的添加 工作, 在齿根区域的网格节点数目应与采样离散点 的数目一致。考虑直齿轮实体模型是由齿廓方程生 成的, 沿齿廓方向各点的受力状态基本相同。因此 在建立齿轮有限元模型时将表 1 直齿轮参数的齿宽 设定为与采样面积宽度一致的 $1 \mathrm{~mm}$, 大大降低重复
的网格数量。此外, 为了验证齿宽为 $1 \mathrm{~mm}$ 时齿轮 有限元模型的可靠性, 考虑模型边缘效应等问题, 分别对齿宽为 $1 \mathrm{~mm} 、 5 \mathrm{~mm} 、 10 \mathrm{~mm}$ 的有限元模型 进行仿真, 保证其他边界、材料等条件相同, 载荷 扭矩分别为 $T 、 5 T 、 10 T$ 进行仿真, 并对仿真结果 进行对比分析, 仿真结果表明三种齿宽条件下齿根 弯曲应力的最大值相差在 5\%以内, 证实 $1 \mathrm{~mm}$ 齿宽 条件下齿轮模型的可靠性。其次将齿轮切分成两部 分, 一部分包含齿根危险区域, 需要进行网格细化, 另一部分不需要调整网格节点, 因此, 网格数量不 做要求。切分操作在 CATIA 中完成, 具体切分过程 如图 2 所示。

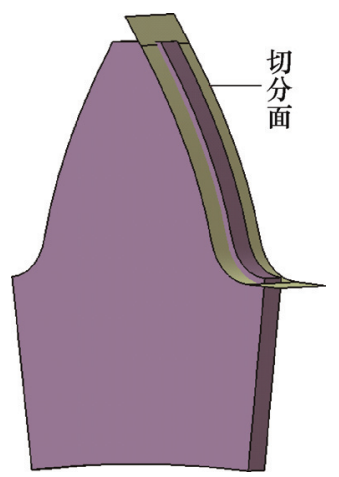

图 2 齿轮切分示意图

将切分后的齿轮两部分分别导入 Hypermesh 中 进行网格划分。对齿根危险区域网格进行局部细化, 齿根细化区域网格尺寸为 $10 \mu \mathrm{m} \times 10 \mu \mathrm{m}$, 细化层数 为六层。同时, 为保证网格质量, 在齿轮啮合区域 网格与齿根危险区域网格的尺寸相差不宜过大, 未 包含齿根危险区域部分齿轮对仿真过程关注的弯曲 应力无影响, 网格尺寸可尽量调大, 最后划分得到 有限元网格数约为 25 万, 直齿轮的网格划分模型如 图 3 所示。

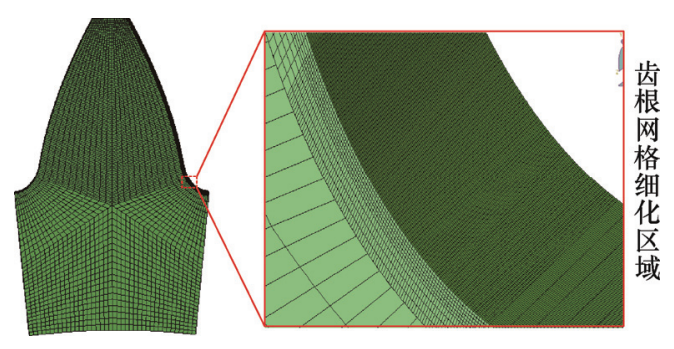

图 3 齿轮网格划分

\section{2 齿根危险区域表面粗粮形貌的生成}

将 Hypermesh 划分好的齿轮模型保存为 inp 文 件导入 ABAQUS 中, 借助 Python 对 ABAQUS 进行 二次开发实现齿根粗粘形貌的添加, 具体操作步骤 如下所示。

(1) 删除直齿轮有限元模型中非齿根细化区域 
网格, 保留齿根网格细化区域。

（2）对齿根网格细化区域表层创建节点集，创 建节点集后删除表层网格选择第二层网格表面创建 节点集, 依次创建不同层数的节点集, 此处论文创 建六层节点集。

(3) 利用 Python 对 ABAQUS 进行二次开发, 提取步骤(2)中创建的节点集的节点坐标信息;

(4) 由步骤(3)获得的节点坐标信息是杂乱无序 的, 对节点坐标依次按照节点沿齿廓方向以及节点 高度值大小进行排序, 获得规则的网格节点编号以 及对应节点坐标;

（5）基于空间坐标变换原理，将采样离散点高 度信息以垂直于齿根危险区域曲面的方式分别对应 添加到 ABAQUS 提取的节点集中, 即改变相应节 点的坐标值。具体变换过程如图 4 所示。

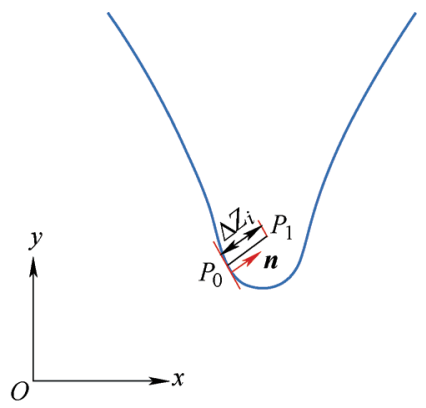

图 4 齿轮网格节点坐标调整示意图

如图 4 所示，直齿轮由齿廓曲线沿齿宽方向拉 伸生成, 因此用平面齿廓曲线代替直齿轮模型。其 中, $P_{0}$ 为齿廓上齿根危险区域的一点, 该点坐标由 步骤(4)获得, $P_{0}$ 点对应曲线上的法向量 $\vec{n}$ 由齿廓方 程得到 ${ }^{[19]}, \Delta Z_{i}$ 为采样离散点高度值, $P_{1}$ 为 $P_{0}$ 沿其 对应法向量 $\boldsymbol{n}$ 方向上添加形貌高度值 $\Delta Z_{i}$ 后的坐 标。其中, 第一层网格节点垂直曲面高度增加值为 $\Delta Z_{i}$ 的 $6 / 6$, 第二层网格节点垂直曲面高度增加值 为 $\Delta Z_{i}$ 的 $5 / 6$, 第三层增加值为 $4 / 6$, 依次类推, 直至离散点高度信息全部添加完成。

（6）将修改完成的网格节点信息替换前面的齿 轮模型 inp 文件对应的网格节点, 至此, 齿根危险 区域表面粗䊁形貌生成。

生成的齿轮形貌示意如图 5 所示。

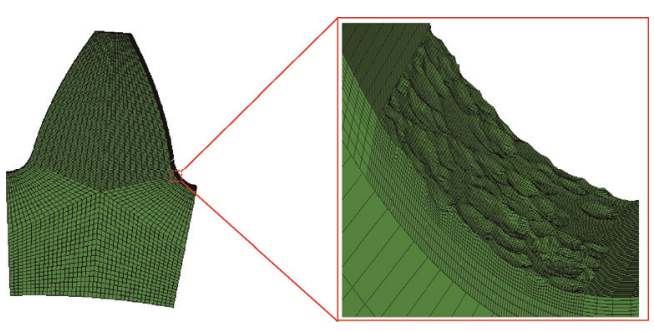

图 5 齿根粗鋉表面有限元模型

\section{3 直齿轮的齿根应力仿真计算}

为了获得粗糙表面参数与应力集中系数的关联 规律, 根据 ISO 25178 标准, 选择其中几个常用的 空间粗糙度表征参数进行计算分析：算术平均高度 $S_{a}$ 、最大谷深 $S_{\mathrm{v}}$ 和十点区域高度 $S_{10 z}$ 。各参数的定 义如下

$$
\begin{gathered}
S_{a}=\frac{1}{m n}\left|\sum_{x=1}^{m} \sum_{y=1}^{n} z(x, y)\right| \\
S_{v}=|\min z(x, y)| \\
S_{10 z}=\frac{1}{5}\left[\sum_{i=1}^{5}\left(z_{i}\right)_{\max }+\sum_{j=1}^{5}\left|\left(z_{i}\right)_{\min }\right|\right]
\end{gathered}
$$

式中, $z$ 为采集形貌离散点的高度, $i$ 和 $j$ 分别表示 喷丸形貌的凸峰和凹谷。三维粗䊁表面参数 $S_{a} 、 S_{v}$ 、 $S_{10 z}$ 与之对应的二维粗粘表面参数为 $R_{a} 、 R_{v} 、 R_{z}$, 二维粗粗表面参数是在三维粗糙表面上截取一条线 段进行计算, 因此, 二维粗粘表面参数会丢失部分 形貌信息, 为了更完整地研究粗䊁表面产生的应力 集中, 论文选择三维粗糙表面参数进行形貌表征。

完成齿根危险区域添加表面粗䊁度的工作后, 论文选择三组齿轮副的啮合模型作为研究对象, 其 中小齿轮的中间齿轮为添加粗糙度的齿轮, 两边齿 轮则未添加。建立耦合和接触对, 将切分的包含粗 楉度的中间齿轮用绑定约束进行连接, 添加载荷并 设置约束条件, 采用准静态分析方法进行仿真分析, 齿轮对的有限元模型如图 6 所示。

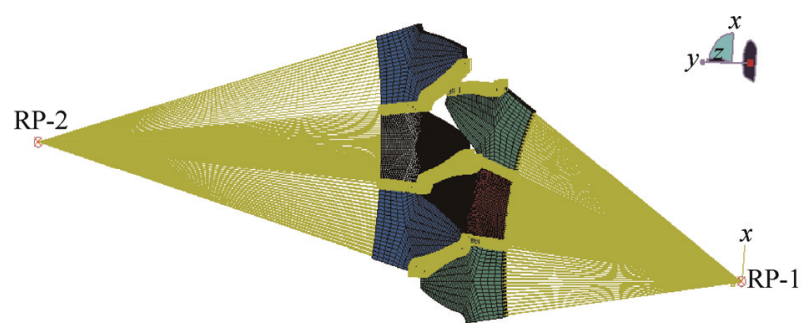

图 6 齿轮对啮合的 3 齿模型

为了研究齿根危险区域表面粗楉度与应力集中 系数的关联规律, 本文采用的应力集中系数定义如 下

$$
K_{t}=\sigma_{\max } / \sigma_{n}
$$

式中, $\sigma_{\text {max }}$ 表示带有缺口模型的缺口处应力最大值, $\sigma_{n}$ 表示光滑试样无缺口的名义应力。此处取 $\sigma_{\text {max }}$ 为 添加粗粗度齿轮模型齿根弯曲应力最大值, $\sigma_{n}$ 为无 粗糙度时齿根弯曲应力最大值。由该定义可知, 通 过两者的比值可消除 $1 \mathrm{~mm}$ 齿宽与实际齿宽不同带 来的影响。

将添加粗糙度的齿轮仿真得到的齿根弯曲应力 
最大值与未添加粗粘度的齿轮弯曲应力最大值相 比, 即得到该粗楉表面参数下对应的应力集中系数 $K_{t}$ 。有无粗粘度的齿轮弯曲应力分布对比如图 7 所 示。为了更清晰地看出齿根粗粘度表面的应力分布, 只选取齿轮切分包含齿根危险区域部分的进行 展示。

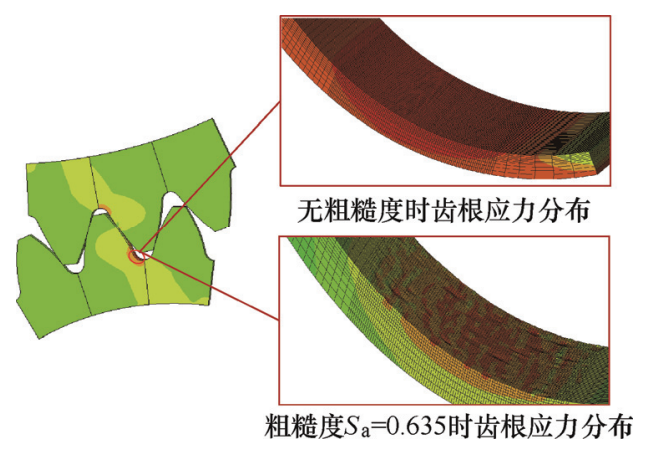

图 7 齿根表面应力分布

如图 7 所示, 带有表面粗糙形貌的齿根应力分 布与无粗粘度时的齿根应力分布相差较大, 粗糙表 面的齿根应力集中主要分布在表面形貌的波谷处, 符合实际情况。取 8 个形貌样本进行仿真分析, 得 到各粗楉表面参数与应力集中系数之间的仿真结果 如表 2 所示。

\section{表 2 不同表面形貌粗粮表面参数 $(\mu \mathrm{m})$ 及其应力集中系数}

\begin{tabular}{ccccc}
\hline 样本 & $S_{a}$ & $S_{10 z}$ & $S_{v}$ & $K_{t}$ \\
\hline 1 & 0.253 & 3.857 & 2.735 & 1.084 \\
2 & 0.361 & 8.692 & 5.731 & 1.151 \\
3 & 0.493 & 6.994 & 5.448 & 1.090 \\
4 & 0.635 & 5.496 & 2.974 & 1.088 \\
5 & 0.925 & 6.590 & 4.097 & 1.090 \\
6 & 1.072 & 7.434 & 4.041 & 1.106 \\
7 & 1.291 & 8.741 & 5.026 & 1.106 \\
8 & 1.415 & 11.228 & 8.299 & 1.144 \\
\hline
\end{tabular}

3 粗粘表面参数与齿根应力集中系数 关联规律

不同于现有对平面粗粘形貌应力集中研究的计 算方法, 论文提出了一种考虑齿根过渡曲线宏观几 何结构上的微观形貌产生的应力集中求解方法。为 了验证论文所研究的求解方法, 将论文的仿真结果 与 $\mathrm{GB} / \mathrm{T} 3480-1997$ 中的相对齿根表面状况系数 $Y_{\text {RrelT }}$ (通过试验获得)进行比较。

在 GB/T 3480-1997 中, 齿根表面状况系数的 定义是 “考虑齿轮根部的表面状况, 主要是齿根圆
角(即齿根危险区域)处的表面粗糙度对齿根弯曲强 度的影响”, 齿根表面状况系数即为齿根粗糙表面产 生的应力集中系数。而相对齿根表面状况系数定义 是 “所计算齿轮的齿根表面状况系数与试验齿轮的 齿根表面状况系数的比值”，给出的计算经验公式 是 ${ }^{[14]}$

$$
Y_{\text {RrelT }}=1.674-0.529\left(R_{z}+1\right)^{0.1}
$$

式中, $R_{z}$ 为二维粗精表面参数中的微观不平度十点 高度, $R_{z}$ 对应论文中的十点区域高度 $S_{10 z}$, 基于 $S_{10 z}$ 的相对齿根表面状况系数公式为

$$
Y_{\text {RrelT }}=1.674-0.529\left(S_{10 z}+1\right)^{0.1}
$$

论文仿真计算的表面粗糙度产生的应力集中系 数(齿根表面状况系数), 与相对齿根表面状况系数 的定义不同, 因此, 不能直接将粗糙表面参数代入 求解, 需要进行转换计算, 转换计算的原理方法如 下: 将国标中定义的试验齿轮 (粗粘表面参数 $S_{10 z}=11.27 \mu \mathrm{m}$ )对应的应力集中系数除以所需要计 算齿轮的应力集中系数即为该计算齿轮的相对齿根 表面状况系数。试验齿轮的粗䊁表面参数 $S_{10 \mathrm{z}}$ 与样 本 8 基本相同。因此, 应力集中系数按样本 8 数据 进行计算, 经转换计算, 表 2 中各粗粘度对应的仿 真计算获得的相对齿根表面状况系数为样本 8 的应 力集中系数除以对应样本的应力集中系数。将各个 样本对应的十点区域高度 $S_{10 z}$ 代入式(6)中, 即得 到国标经验公式所计算的 $Y_{\text {RrelT }}$ 值。对比结果如图 8 所示。

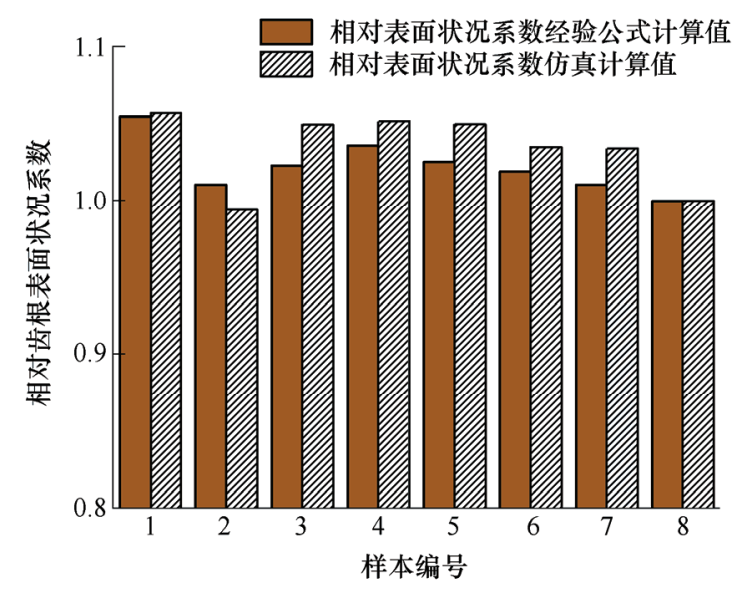

图 8 相对齿根表面状况系数对比

如图 8 所示, 仿真计算得到的相对齿根表面状 况系数与国标经验公式计算得到的系数整体趋势相 同, 且两者计算得到的系数值也十分接近。此外, 在国标中注释了经过强化处理(如喷丸)的齿轮, 其 相对齿根表面状况系数要稍微大于计算经验公式所 确定的数值, 从图 8 整体趋势不难看出仿真值稍微 
大于经验公式计算的数值, 与国标中的注释说法一 致。图 8 从侧面论证了论文仿真求解方法的可信度。 此外, 国标经验公式间接表示了齿轮齿根处形貌引 起的应力集中, 而本文则直接求解对应形貌的应力 集中, 表现更加直观、具体。此外, 与实验获得应 力集中时耗时耗力相比, 通过论文可以快速、低成 本计算出应力集中系数, 为后续精确计算齿轮弯曲 疲劳寿命提供技术支持。

为了得到喷丸表面三维粗糙表面参数与应力集 中系数的关联规律, 借助 Matlab 软件的 cftool 工具 包对表 2 中的数据进行关系式拟合, 得到拟合关系 如图 9 所示。

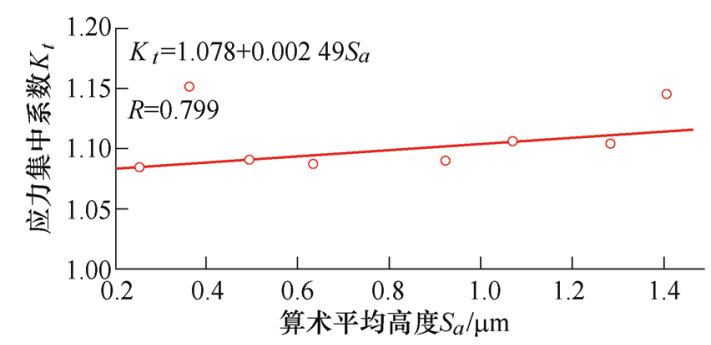

(a) $K_{t}$ 与 $S_{a}$ 的关系曲线图

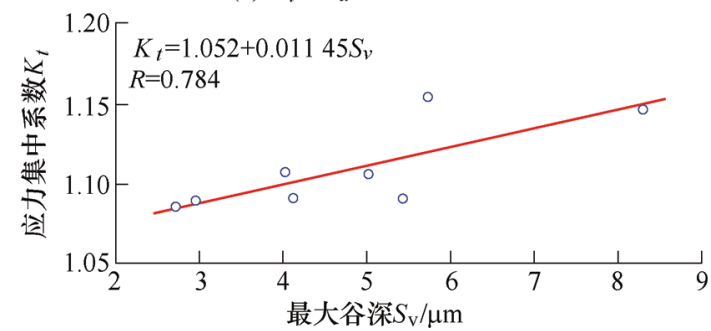

(b) $K_{t}$ 与 $S_{v}$ 的关系曲线区

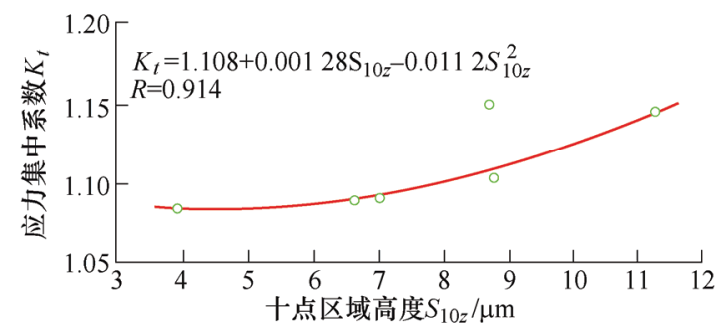

(c) $K_{t}$ 与 $S_{10 z}$ 的关系曲线区

图 9 应力集中系数与各粗粘表面参数的拟合曲线图

图 9a、9b、9c 分别表示粗粘表面参数 $S_{a} 、 S_{v}$ 、 $S_{10 z}$ 与应力集中系数 $K_{t}$ 的拟合曲线图, 对应的拟合 相关系数 $R$ 分别为 $0.799,0.784,0.914$ 。在对 $K_{t}$ 的 拟合关系中, 十点区域高度 $S_{10 z}$ 的拟合效果最好, 拟合公式如式(7)所示

$$
K_{t}=1.108+0.00128 S_{10 z}-0.0112 S_{10 z}{ }^{2}
$$

式(7)的拟合公式可以较好表征粗粮表面形貌 与应力集中系数的定量关系。需要说明的是, 加工 零部件图纸上标注的粗䊁表面参数 $R a$ 是粗粮表面 的二维表征参数, 表征三维粗楉表面具有一定的局
限性, 选择三维粗楉表面参数更为合适。从论文的 分析结果看, 为更加科学的表征三维粗精表面对应 力集中的影响, 使用十点区域高度参数 $S_{10 z}$ 更加科 学, 工程应用中, 可优先使用 $S_{10 \mathrm{z}}$ 对应力集中系数 进行计算。

\section{4 结论}

（1）本文提出了一种基于齿根三维粗䊁表面形 貌的有限元建模与分析方法, 解决了三维粗粘齿根 表面应力集中系数计算问题, 为齿轮表面完整性与 抗疲劳设计研究提供了一种基础方法。

（2）论文采用有限元计算得到的表面粗糙度引 起的应力集中系数与 GB/T 3480-1997 中给出的相 对齿根表面状况系数经验公式进行间接对比，两者 计算结果基本相同。

(3) 对有限元计算得到的数据进行拟合分析, 结果表明粗䊁表面参数 $S_{a} 、 S_{v} 、 S_{10 z}$ 拟合应力集中 系数的拟合公式的相关系数分别为 $0.799,0.784$, 0.914 , 十点区域高度参数 $S_{10 z}$ 能较好地表征齿根表 面的应力集中, 工程应用中可用十点区域高度 $S_{10 z}$ 对喷丸粗粘表面产生的应力集中进行计算与表征。

\section{参 考 文 献}

[1] AROLA D, WILLIAMS C L. Surface texture, fatigue, and the reduction in stiffness of fiber reinforced plastics[J]. Journal of Engineering Materials \& Technology, 2002, 124(2): $160-166$.

[2] NEUBER H. Theory of notch stresses[M]. Berlin Verlag, 1958.

[3] AROLA D, WILLIAMS C L. Estimating the fatigue stress concentration factor of machined surfaces[J]. International Journal of Fatigue， 2002， 24(9): 923-930.

[4] CHAN, KWAI S. Characterization and analysis of surface notches on Ti-alloy plates fabricated by additive manufacturing techniques[J]. Surface Topography Metrology \& Properties, 2015, 3(4): 044006.

[5] KANTZOS C, LAO J, ROLLETT A D, et al. Design of an interpretable convolutional neural network for stress concentration prediction in rough surfaces[J]. Materials Characterization, 2019, 158: 109961.

[6] MEDINA H , HINDERLITER B R. The stress concentration factor for slightly roughened random surfaces: Analytical solution[J]. International Journal of Solids and Structures, 2014, 51(10): 2012-2018. 
[7] MEDINA H. A stress-concentration-formula generating equation for arbitrary shallow surfaces[J]. International Journal of Solids and Structures，2015，69-70: 86-93.

[8] CHENG Zhengkun, LU Wei, LIAO Ridong, et al. Surface stress concentration factor via Fourier representation and its application for machined surfaces[J]. International Journal of Solids and Structures, 2017, 113-114: 108-117.

[9] CHENG Zhengkun, Lu Wei, LIAO Ridong, et al. Fatigue notch factors prediction of rough specimen by the theory of critical distance[J]. International Journal of Fatigue, 2017, 104: 195-205.

[10] CHENG Zhengkun, LIAO Ridong. Effect of surface topography on stress concentration factor[J]. Chinese Journal of Mechanical Engineering, 2015, 28(6) : 1141-1148.

[11] MEREUTA V, BUCIUMEANU M, PALAGHIAN L. 3D roughness parameters as factors in determining the evolution of effective stress concentration factors in fatigue processes[J]. Applied Mechanics \& Materials, 2012, 248: 504-510.

[12] LI Guowen, TANG Jinyuan, ZHOU Wei, et al. Fatigue life prediction of workpiece with 3D rough surface topography based on surface reconstruction technology[J]. Journal of Central South University, 2018, 25(9): 2069-2075.

[13] XU Shanhua, WANG Youde. Estimating the effects of corrosion pits on the fatigue life of steel plate based on the 3D profile[J]. International Journal of Fatigue, 2015, 72: $27-41$.

[14] 国家技术监督局. GB/T 3480-1997 渐开线圆柱齿轮承 载能力计算方法[S]. 北京: 中国标准出版社, 1997.

State Bureau of Technical Supervision. GB / T 3480-1997 calculation method for load bearing capacity of involute cylindrical gears[S]. Beijing: China Standard Press, 1997.

[15] 刘忠明, 袁玉鹏, 肖伟中, 等. 大模数齿条齿根应力计 算方法研究及测试[J]. 机械工程学报, 2016, 52(23): 152-159.
LIU Zhongming, YUAN Yupeng, XIAO Weizhong, et al. Research and test of large modulus rack root stress calculation method[J]. Journal of mechanical engineering, 2016, 52(23): 152-159.

[16] 胡维平, 杜明辉. 信号采样率对经验模态分解的影响研 究[J]. 信号处理, 2007(4): 637-640.

HU Weiping, DU Minghui. The influence of signal sampling rate on empirical mode decomposition[J]. Signal Processing, 2007 (4): 637-640.

[17] 陈兵奎, 高艳娥, 梁栋. 共轭曲线齿轮齿面的构建 $[\mathrm{J}]$. 机械工程学报, 2014, 50(3): 18-24.

CHEN Bingkui, GAO Yan'e, LIANG Dong. Construction of conjugate curve gear tooth surface[J]. Journal of Mechanical Engineering, 2014，50(3): 18-24.

[18] 陈兵奎, 梁栋, 高艳娥. 齿轮传动共轭曲线原理 [J]. 机 械工程学报，2014，50(1)：130-136.

CHEN Bingkui, LIANG Dong, GAO Yane. Principle of conjugate curve of gear transmission[J]. Journal of Mechanical Engineering, 2014, 50 (1): 130-136.

[19] 唐进元, 周长江, 吴运新. 齿轮弯曲强度有限元分析精 确建模的探讨 [J]. 机械科学与技术, 2004(10) : 1146-1149, 1248.

TANG Jinyuan, ZHOU Changjiang, WU Yunxin. Discussion on accurate modeling of finite element analysis of gear bending strength[J]. Mechanical Science and Technology, 2004(10): 1146-1149, 1248.

[20] 张质子, 唐进元. 多条平面曲线数值合成新方法 [J]. 机 械传动, 2016，40(9): 167-169.

ZHANG Zhizi, TANG Jinyuan. A new method for numerical synthesis of multiple plane curves[J]. Mechanical Transmission, 2016, 40(9): 167-169.

作者简介: 何玉辉, 男, 1974 年出生, 博士, 副教授。主要研究方向为 超声振动切削加工原理、工艺及设备。

E-mail: csuhyh@163.com

唐进元(通信作者), 男, 1962 年出生, 教授, 博士研究生导师。主要研 究方向为复杂曲面宏微观形貌设计制造、数控机床与数字化制造与检测。

E-mail: jytangcsu@163.com 